\title{
Glomerulonefritis Membranoproliferativa (GNMP). Correlaciones Clínico - Patológicas y Terapéuticas
}

\author{
Dr. Rodrigo Urizar E. ${ }^{1}$, Dr. Jorge Cerda 2
}

Membranoproliferative Glomerulonephritis (MPGN)

\begin{abstract}
Membranoproliterative glomerulonepluitis is a chronic progressive entity leading to end stage renal disease in greater than $50 \%$ of cases after 5 years of diagnosis. Clinically, it often presents insidiously with homaturia-protet. nuria, hypocomplementernis, nephrotic syndrome, arterial hypertension or combinations thereof. Acute and progressive renal failure may also occur. UItrastructurally, type l shows primarily intramesangial and subendothelial deposits whereas type 2 displays a partial or complete replacement of the lamina densa by a denser deposit, hence, the name of dense deposit disease. Greater than $80 \%$ of MPGN 2 patients show a circulating autoantibody (an abnormal $\mathrm{IgG}, \mathrm{C}_{3} \mathrm{Nef}$ ) which blocks the $\mathrm{C}_{3}$ convertase inactivator of the alternative pathway of complement activation whereas in type I MPGI hypocomplementemia may be mediated by activation of the classical pathway. Circulating or "in situ" immune complex (IC) mediation and thrombocytopathic mechanisms seem to play major roles in producing, maintaining and enhancing progression of inflammation and sclerosis that leads to end stage renal disease. Thus, decteased ptatelet survival, platelet antigen deposition in glomerular capillaries and platelet release of myointimal histiocyte and mesangial coll growth factors have been demonstrated The edrly treatment of these abnormalities has, for the first time, demonstrated in a consistent way, that the use of prednisone increases significantly the patient survival. More recently, similar results have been reported in a prospective double blind placebo control study using aspisin and dipyridamole in patients with MPGN type 1 only. Prednisone, aspirin and dipyridamole are potent antiplatelet aggregat in and antiplatelet release agents Thus, it stands to reason, and it is strongly recommended to initiate early troatment of both types of this entity, or for that matter of any other progressive and sclerosing glomerulopathy, with a combination of these 3 substances given for a prolonged period of time. It is these clinicians' conviction that this form of therapy is fully indicated in MPGN regardless of the histopathology and that therapeutic inertia is no longer justifiable.

(Key words: Membrano proliferative Gomcrulonephritis. Inmune Complex. Trombocytopathic Mechanism. Treatment. Aspirin. Dipyridunole. Prednisone).
\end{abstract}

El sindrome nefrítico crónico-progresivo, produce insuficiencia renal terminal (IRT) en un número importante de pacientes ${ }^{17}$. Una entidad representativa de este proceso, la GNMP, ha sido descrita recientemente por Gotoff4s y West $y$ colaboradores ${ }^{106}$ : quienes definieron las bases clínico-patológicas de esta nefropatía. Las caracteristicas serológicas, inmunohistopatológicas y ultraestructurales permiten distinguir claramente a la GNMP de otras glomerulopatias agudas o crónicas (Tabla 1). La presencia de hipocomplementemia persistente y la microscopia elec-

1. New York State Department of Health and Abany Medical College, Division of Pediatric Nephrology Albany, New York, USA (Urizar).

2. Departamento de Fisiología - Centro de Nefrología. Facultad de Medicina, Montevideo, Uruguay (Cerdá). trónica diferencian dos variedades fundamentales: Los depósitos electrónicos nodulares, granulares y extendidos (agregados) localizados en el subepitelio, subendotelio y dentro de la matriz mesángica idehtifican a la GNMP de Tipo 1. En el Tipo 2, se encuentra un depósito de densidad mayor $y$ de composición química ligeramente distinta de la membrana basal glomerular (mbg) normal $7,42,48,56,58,59,65$, a la cual reemplaza parcial o completamente (distribución segmentaria o difusa) $)^{5,59}$. Este depósito se observa también en la membrana basal de la cápsula de Bowman y de los túbulos renales. Cabe destacar que los depósitos subepiteliales y subendoteliales coexisten frecuentemente en ambos tipos de GNMP. Por lo tanto, su presencia y localización no proporcionarían un criterio histopatológico definitivo para diferenciar una tercera variedad, la GNMP de Tipo $3^{3-31}$, 
92-95, que se caracterizaría por presentar una mezcla de depósitos granulares epimembranosos, subendoteliales, mesángicos e intramembranosos. Por otra parte, la mbg que generalmente se mantiene intacta en el Tipo 1, presenta laminación y duplicación en el Tipo 2; mientras que en el Tipo 3, las alteraciones de la mbg se asociarían a depósitos epimembranosos juxtapuestos a depósitos densos subendoteliales ${ }^{48}, 92-94$. Sin embargo, por las razones mencionadas antes, la variedad 3 no es aceptada universalmente como una forma individual de esta nefropatia sino que se la consideraría parte del espectro histopatológico de los dos tipos fundamentales. Swainson y colaboradores ${ }^{95}$ han propuesto que kos casos con depósitos epimembranosos y subendoteliales y con alteraciones significativas de la mbg descritos por Strife y colaboradores ${ }^{94}$ sean subclasificados como GNMP Tipo I a.

En la microscopia de luz se destacan el aumento de tamaño y la lobulación del glomérulo. La proliferación y expansión periférica de] mesangio (células y matriz) resultan en la interposición de este entre la mbg y el endotelio. La impregnación argéntica de secciones finas, permite apreciar claramente este fenómeno que constituye el doble contorno ("tram track" o "rieles de tranvia") parietal periférico característico de la GNMP Tipo 1. Lesiones similares pero debidas a otros mecanismos que no deben confundirse con las descritas se observan mediante inmunofluorescencia en la variedad 2. Las glomerulonefritis semcjantes a la forma membranoproliferativa Tipo 1 se encuentran en pacientes bacterémicos con endocarditis subaguda, con vasculitis, con infecciones virales e infestaciones parasitarias o en procesos de coagulación intravascular con compromiso renal. La inmuno. fluorescencia ha sido resumida en la Tabla 1. Cabe destacar que los depósitos importantes de $\mathrm{C}_{3}$ aislado o acompañado de inmunoglobulinas se encuentran en ambos tipos de GNMP; contornos dobles producidos por la localización de $\mathrm{C}_{3}$ en las porciones periféricas de la mbg pero no en el depósito denso y los anillos intramesángicos $\left(\mathrm{C}_{3}\right.$ alrededor de depósitos mesangiales) se han descrito sólo en el Tipo 258,59. Ejemplos de la inmunohistopatologia y ultracstructura renal de la G.NMP se muestran en las Figuras 1 a 4.

La sintonimia de esta enfermedad es múltiple e incluye denominaciones tales como: GN hipocomplementémica crónica y lobular, $\mathrm{GN}$ mixta membranoproliferativa, $G N$ parietoproliferativa, GN mesangioproliferativa crónica y $G N$ mesangiocapilar, La frecuencia de la GNMP no se conoce con exactitud y varía, en las series publicadas, entre el $5 \%$ de todas las biopsias renales realizadas en afgunos servicios hasta un $22 \%$ de todas las glomerulo patias $2,21,46-48,85,92$, 110,111 . Nuestra impresión personal es, sin cmbargo, que esta entidad presenta ciertas preferencias geográficas y que su número es probablemente mayor que lo estimado alcanzando ta! vez a más de un $25 \%$ de las glomerulonefritis crónicas activas en la población pediátrica. En centros europeos particularmente en Francia se ha notado una disminución del número de casos de GNMP diagnosticados en los últimos años (Dr. Renée Habib, comuniçación perşonal).

Si bien la histopatologia característica permite la separación. de los grupos fundamentales, no existe una presentación y/o manifestaciones clínicas típicas de una u otra forma de GNMP. Se detectan algunas diferencias tempranas sin embargo, en relación a la edad de los pacientes, sin predominio de un sexo sobre el otro. Nos permi-

Tabla 1.

Alteraciones inmunohistopatológicas, Lltraestructurales y del Complemento Sérico Observadas en la Glomerulonefritis Membranoproliferativa

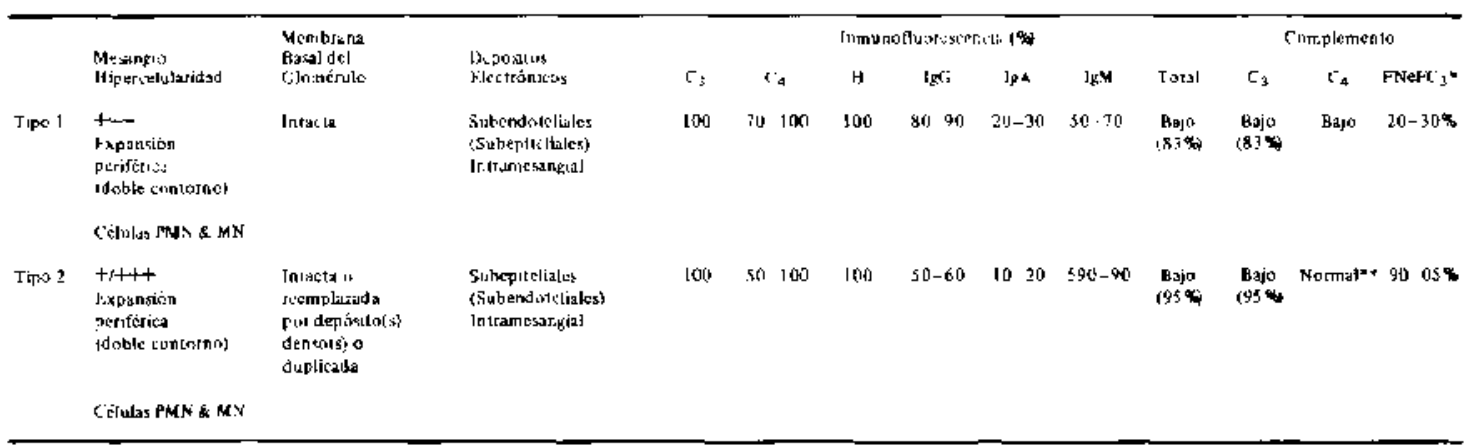

* FineFe ${ }_{3}$, factor nefritiso

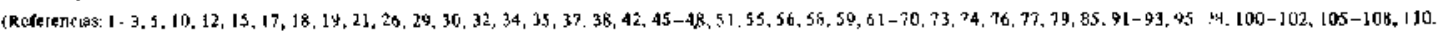
$115,117,118 \%$ 
tımos insistir en la gran importancia clínica de los casos que muestran sindrome nefrótico o sinto. mas y signos similares a una $G N$ post estrepto. cócica (GNDA), en que el paciente ocasional presentat evidencia directa o indirecta de infec. ción estreptocócica reciente, reforzando el diag. nóstico de GND $\Lambda$. En estos casos, en el periodo en que los síntomas de la nefrit is aguda post infecciosa debieran desaparecer por completo ( 4 a 6 semanas) aún se encuentran alteraciones urinarias o hipocomplementenia persistentes. Lid mayoría de los pacientes con GNMP presentan la triada compuesta de hematuria, proteinuria $y$ valores disminuidos de $C_{3}$ sanguíneo. Algunos enfermos demuestran progresión rápida hacia la IRT mientras que otros son referidos al pediatra nefrólogo porque en el curso de le evaluación de una proteinuria -hematuria persistente, de anemia $a$ de hipertensión arterial, se detecta una elevación de la credtisina sérica en presencia de hipotomplementemia mantenida. Se consideran elementos de mal pronóstico en pacientes jóvencs con GNMP, el dcsarrollo o la presentación con síndrome nefrótico, el compromiso precoz de la función renal, concentraciones elevadas de factor nefrítico $\left(C_{3} \mathrm{NeF}\right)$ sérico y abundantes semilunas epiteljales ya sea en la biopsia inicial o en cualesquiera otros períodos del curso de la en. fermedad.

Se evidencia por lo tanto, que el impacto clínico de la GNMP está dado fundamentalmente por su evolución que, a pesar de ser prolongada, llcva irreversiblementc a la insuficiencia renal a la gran mayoria de $\operatorname{los} \operatorname{casos}^{19}, 30,34,45,47,58$, $60,67,70$, entre 3 a 11 años después del contienzo de la enfermedad. A este respecto, las publicaciones de Anloine, Camerón, Davis. Habib y Swainson $2,19,30,46,47,95$ indican que 5 a 10 años del diagnóstico de la GNMP, 30 a $50 \%$ de los pacientes presentaban IRT.
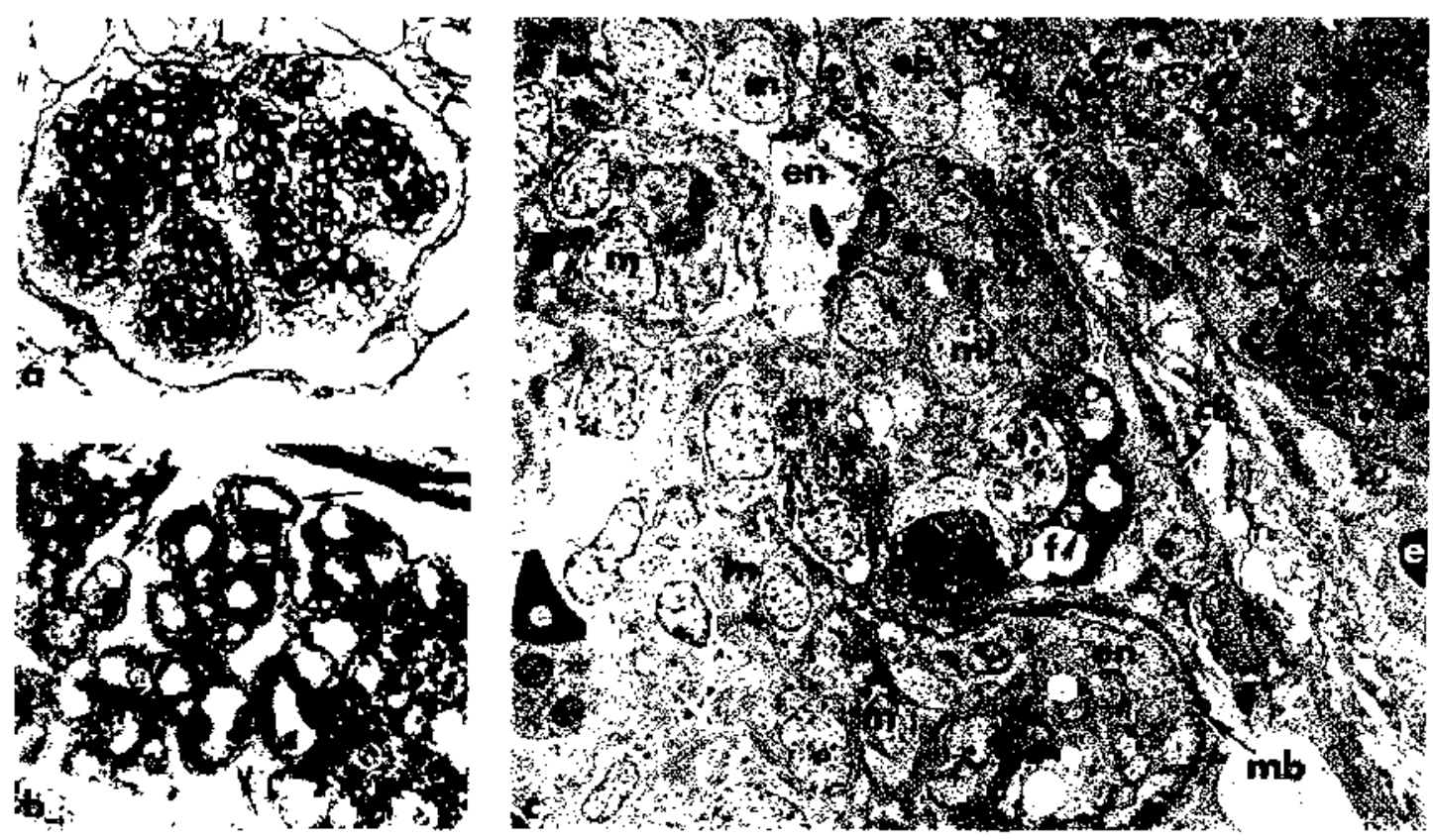

Figura 1. Composición microfolográfica de un pacience con GNMP 1.

a. Microscopía de luz que demuestra aumento del tamaño y lobulación glomerular debido a hiperplasia mesángica. La petiferia de cada lóbulo represcnta un "contorno doble" que se observa también en algunas asas internas $y$ en la Figura $1 \mathrm{~b} ; 250 \mathrm{X}$; metornamina argéntica.

b. Abundantes "contornos dobles" ("railroad track sign") se destacan claramente (flechas) niediante la impregnación argéntica: $500 \mathrm{X}$.

c. Microfotografía electrónica de bajo aumento en que se obscrya proliferación celular $y$ aumento de la matriz $\mathrm{y}$ de las células mesángicas $(\mathrm{m})$. Los pedicclios están parcialmente fusionados, hay abundances depósitos subendoteliales e intramesángicos y aflujo de PMN (asteriscos). En el espacio subendotelial del capjlar central y hacia la derecha del PMN, se observan numerosos depósitos de mayor densidad electrónica (flecha) que corresponden a acúmulos de fibrinógeno-fibrina $(\mathbf{0} ; \mathrm{mb}$, membrana basal; en, endotelio; e (blanca), eritrocito en vénula intersticial (deiecha) y lumen capilar glonerular (izqujerda); e (negra), epitelio parietai; cB, cápsula de Bowman; op, epitelio visceral; tp, túbulo proximal. Glutaraldehjdo, acetato de uranilo-citrato plúmbico, $4000 X$. 


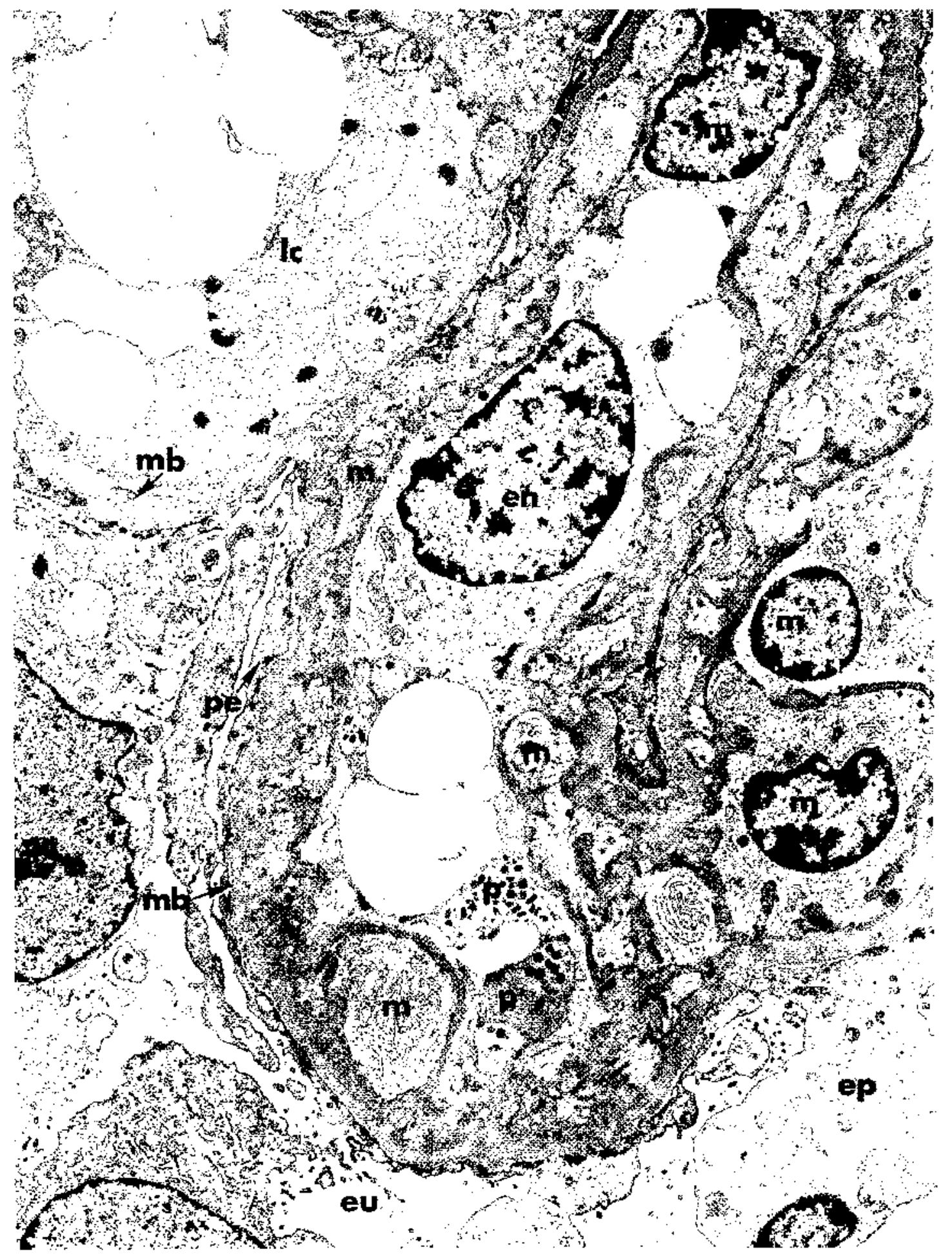

Figura 2. Microfotografía clectrónica de un paciente con GNMP 1, et que se obserya a mayor aumento las alteraciones ultraestructurales descritas en la Figura 1 . Nótese la interposición y expansión periféricá del mesangio(m) crtre la membrana basal capilar(mb) y cl cndotelio(en). Plaquetas(p), en diversos estados de granulación aparecen acumuladas en el bumen capilat. La mb sstá laminada, especialmente en el capilar del ángulo supcrior izquicrdo de la fotografia. Los podicelios(pe) están fusionados y las células epiteliales(ep) presentan edema citoplasmático y abundante retículo e inclusiones. eu, sspacio urinario: en, cndotelio; 1 c, lumen capilar. Ace tato de uranilo, citrato plúmbico, $\mathrm{X} 8000$. 

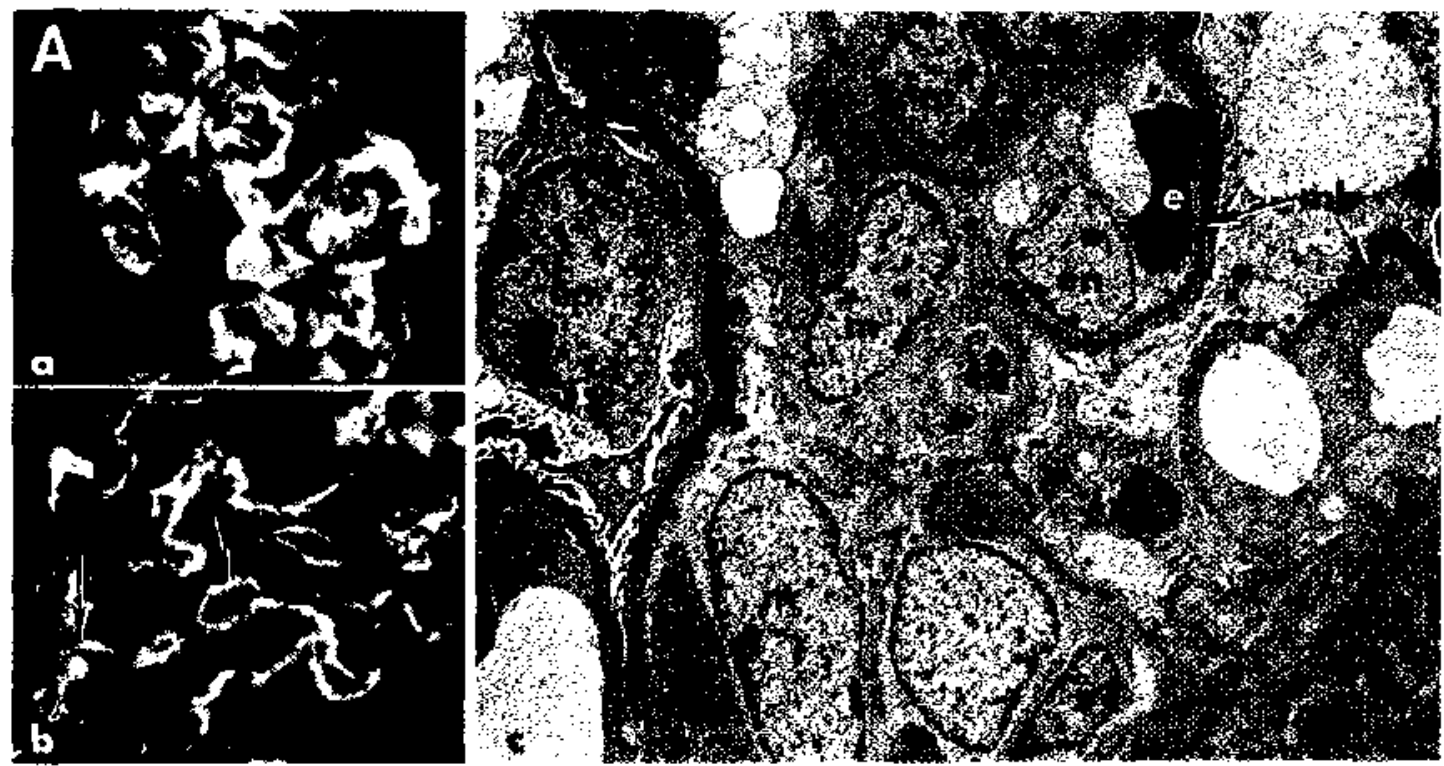

Figura 3. Esta füura esta constituida por las secciones A y B cn las que se contrastan dos subtipon de G.VMP 2.

A,a. Depósitos semilincares y acintados de injunofluorescencia uniformerte positiva se observan a lo larģo de la pared capilar conteniendo gránulos y nódulos ocasionales. Antisuero de conejo anti $\mathrm{C}_{3}$ ìmmano. $250 \mathrm{X}$.

b. Depósitos predominantementc gtanulares (flcchas) se muestran en esta microlotografía con intisuers de concjo contra lgG humana, en el misno paciente ilustrado en a y c. $280 \mathrm{X}$.

c. Mícrolotogratía electrónica en la que se destaca el reemplazo total de li metnhrana básal(mb) por ur depósito denso caractetistico de la GNMP 2 (subtipo difuso). Pequeñas jnterupeiones de este material y ja presencia de depósitos subepiteliales se señalan mediante asteriscos pequeños que también indican depósitoss intranesángitos (ángulo inferior derecho). Los asteriscos de mayor tamalso muestran leucocitos PMN, uno de los cualcs aparece en el espacio uritario. m, mesangio: en, endotelio; $\mathrm{cp}$, epitelio tn que se observa tusión de los pedicetios: c, critrocito en lumen capilar. Aceto de ưauile, citrato plúmbico, $5000 \mathrm{X}$, Este paciente ptesentó lęmaturia, proteinuria progrestva $y$ síndrome nefrótico sin comptoniso de la función renal $y$ sin desarrollo de semilunas epitcliales. La biopsia fuc practicada a los 3 neses de conocidas las alteraciones urinarias y de hipocomplementemia: la paciente recibió trutauicuto combinado (Prednisonal y dipiridamols por 4 años.
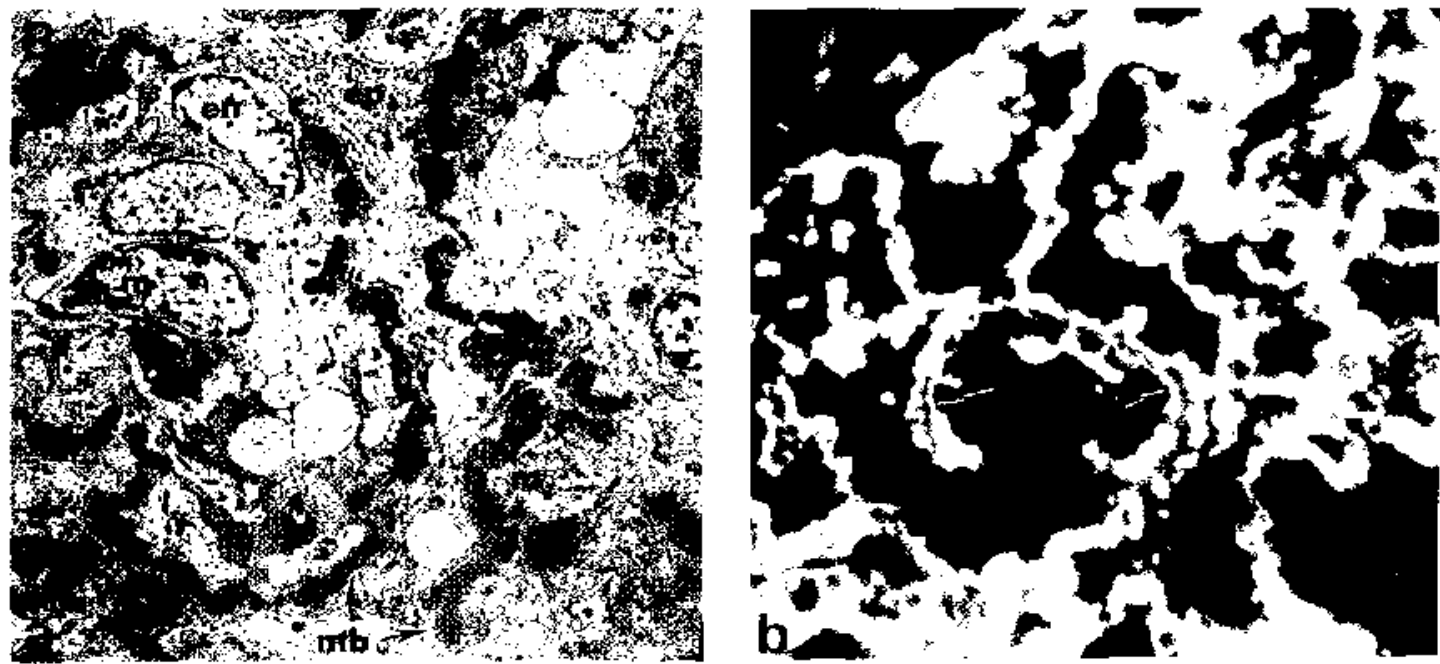

B,a. Esta mier ofotografía electrónica muestra depósitus antiguos densus in tramembranosos, de mayor grosor (distribución segmentaria, ststeriscos) que aquélos que aparccen en la Figura $3, \mathrm{~A}$, c; se observan además a lgunos depósitos intrame sángicos (asterisco). mb, mumbrana basal; ep, epitelio; in, mesangio; tn, endotelio. 4500X.

b. I a inmunofluorescencia indica claramente la localización, distribución, tajaño y grada de agreganiento de los depósitos de C.3, puestos én evjđencia mediante antisuero de conejo contra $C_{3}$ humano. $1200 \mathrm{X}$

Paciente que presentó síndrome nefrótico iodiopático resistente a prednisona y que posteriomente demostró hipocomplementemia mantenida $y$ hematuria. Biopsia renal practicada a los 3 afjos de iniciada la cnfermedad. 


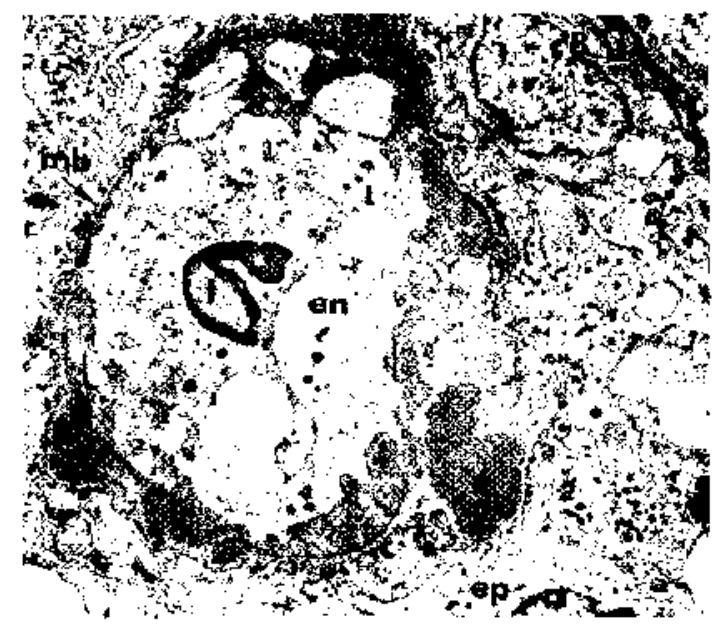

Figura 4. Micrografía efectrónica de un paciente con GNMP I. Se obstran depósitos claramente delineados (asteriscos) en ambos lalos de la menbrana basal(mb), la clue aparece ateluda y parcialmente laminada. Fstiss alteraciones correspomderáan a la CNMP 3 que no es unjversulmente aceptada como ifna entidad septrada de lus tipos l y 2 . I (Véas textol. I, Iencocilo polinorionuclear; en, endotclio: ep, epitclio; $c B$, cápsula de Bowmian. 6000X.

La etiología de la GNMP se desconoce y su patogenia aun no se ha aclarado complotamente; es muy posible sir embargo que mecanismos inmunológicos tengan un rol importante en el desarrollo y progresión de la inflamación glomerular. La formación de inmunocomplejos (IC) circulantes o"in situ" 17, 30, 31, 51, 52, 64, 70, 71. 80 requiere la participación de elementos amplificadores: del complemento y sus activadores, de las prostaglandinas y de la coagulación ${ }^{13,88}$. Los $\mathbf{I C}$ circulantes no se detectan regularmente en todos los casos de GNMP110 lo que está en desacuerdo con los hallazgos de la microscopía de irrmunofluorescencia que en la gran mayoría de los pacientes demuestra depósitos granulares o nodulares que presumiblemente representen IC agregados localizados en el glomérulo. Es plausible sin embargo, que una cantidad variable pero subliminal de IC active los sistemas liumorales-celulares que producen $y$ perpetúan la inflamación (y la esclerosis) glomerular mediante la acción de enzimas proteolíticas producidas por los leucocitos polimorfonucleares (PMN) y mononucleares (MN). Ambos tipos de células, responsables de la necrosis glomerular, se encuentran frecuentemente en la GNMP y en otras glomerulopatías humanas y experimen. tales $^{76}$. La activación sistémica del complemento sería un fenómeno importante pero no esencial para el desarrollo de esta enfermedad. Es posible que una vez que la inflamación se pone en marcha, el consumo local de los factores complementarios (depósitos tisulares) mantenga la función flogogénica de este proceso. Esta actividad mínima pero constante de los sistemas de amplifación explicaría algunas de las características de la GNMP: no todos los pacientes presentan hipocomplementemia; los niveles del complemento sérico no se correlacionan en forma estricta con la gravedad y la progresión de las lesiones glomerulares; se encuentran depósitos glomerulares de $\mathrm{C}_{3}$ en las biopsias de todos los pacientes en que se hacen (y en algunos de aquellos que han recibido un trasplante renal por esta enfermedad) independientemente de las concentraciones séricas de $C_{3} 19.117$; la evolución progresiva pero prolongada, refleja probablemente una inflamación de poca intensidad; pacientes con cifras altas de factor nefrítico presentan una evolución clínica más grave y de menor duración que aquellos en que se detecta actividad minima del factor nefrítico. Se desconoce aún el significado real que este factor pueda tener; se sabe sin embargo que es un autoanticuerpo (inmunoglobulina $G$ ) contra la convertasa del $\mathrm{C}_{3}$ activado por la vía alternativa, ${ }^{\text {ag }}$ cuya porción más pesada está constituida por una cadena de mayor longitud que en la IgG normal.

Finalmente, un componente trombocitopático ha sidlo descrito recientemente, y parece tener una inportancia fundamental en la patogenia de la GNMP, habiéndose demostrado, en estos pacientes, disminución de la vida media de las plaquetas $^{13}, 16,44,50$ localización de antígenos plaquetarios en los capilares glomerulares 16.27 . $2 \mathrm{a}, 38,50,74$ y liberación de substancias intraplaquetarias altamente estimulantes del crecimiento y maduración de células endoteliales, musculares, mesángicas e histiocitarias ${ }^{16,27,28}$, $38,50,81,88$. Este último factor derivado de las plaquetas (FDP), seria sintetizado bajo la influencia de macrófagos mononucleares provenientes de la médula ósea y localizados en el gloméru$10^{76}$. Estas células conjuntamente con las plaquetas producen fibronectina que pavimenta los tejidos dañados y estimula la adhesión de histiocitos u otras células y la producción de colágeno. E1 resultado final, esclerosis glomerular progresiva e IRT, se observa cuando la colagerización ha alcanzado un nivel crítico.

La asociación de la GNMP con la deficiencia de antitripsina alfa $1(\mathrm{AT} \alpha \mathrm{l})$ y con la lipodistrofia parcial (LDP) constituye una característica única de esta nefropatía. Se han descrito aproximadamente 7 pacientes con deficiencia de $A T \alpha l^{93}$ que demostraban sintomas y signos de enfermedad hepática, ausencia de daño pulmonar y que desarrollaron GNMP de Tipo 1. Es importante destacar que la bepatopatia esta presente cuando 
se detecta la deficiencia de AT $\alpha 1$, mientras que la GNMP se desarrolla tardíamente. El curso clínico de la nefropatía es independiente de la concentración plasmática de AT $\alpha 1$ que en más del $60 \%$ de los casos es del fenotipo MM o PIZZ; numerosos pacientes de ambos fenotipos sin embargo, no desarrollan glomerulonefritis. Debido a que la GNMP Tipo 1 se asocia con otras enfermedades hepaticas en especial hepatitis crónica activa, es posible que la inflamación glomerular esté relacionada con el daño hepático parenquimatoso o se deba a la antigenemia viral mantenida.

La GNMP de ambos tipos ha sido descrita en pacientes con LDP; casos con lipodistrofia total o de las extremidades asociados con nefritis no se han detectado hasta la fecha. Sissons y colaboradores ${ }^{90}$ en su serie de 25 pacientes con lipodistrofia comprobaron hipocomplementemia en 17 $\left(\mathrm{C}_{3}\right.$ disminuido con niveles normales de $\mathrm{C}_{4} \mathrm{y}$ $\mathrm{C}_{3}$ ) : en 14 de los 17 se detectó factor nefrítico en el suero. Los estudios ultraestructurales demostraron GNMP Tipo 2 en tres pacientes mientras que en otro, la GNMP era del Tipo 1. No fue posible la caracterización histológica precisa en otros 3 pacientes en quienes no se utilizó microscopía electrónica. Sissons y colaboradores $^{90}$ demostraron claramente que la LDP precede al desarrollo de la GNMP en 5 a 20 años; además destacaron que los pacientes con LDP pueden presentar normocomplementemia $y$ ausencia de glomerulopatia e hipocomplementemia sin GNMP (19 casos). Este trabajo indica también que el factor nefrítico detectado en la LDP con o sin GNMP parece ser idèntico a aquel que se encuentra en la GNMP idiopática de Tipo 2 sin LDP. Conviene destacar que la activación mantenida del $C_{3}$ característica de la GNMP Tipo 2 no es única ya que existen otros factores plasmáticos, además de la IgG anormal, que actúan en forma semejante sobre el complemento. Desde el punto de vista histológico no existe diferencia entre la GNMP de pacientes con $y$ sin LDP. Los autores ${ }^{90}$ proponen que la hipocomplementemia conduce a una opsonización deficiente que aumentaría la susceptibilidad del paciente a las infecciones, creando una antigenemia persistente. Permanece aún sin aclarar la asociación entre hipocomplementemia y LDP.

Numerosos regímenes terapéuticos se han utilizado en un esfuerzo por contener el avance progresivo de la GNMP. Debido a la falta de controles adecuados, al uso aislado o combinado de múltiples drogas inmunosupresoras, antiinflamatorias y antiagregantes de las plaquetas $y$ a la irregularidad en la duración de los tratamientos, los resultados han sido variables y de escaso valor estadístico $2,23,24,30,46-48,66,96.5$ in embargo, a pesar de la falta de estudios al azar y prospectivos, se aprecia claramente un efecto favorable de los antiagregantes plaquetarios (aspirina y dipiridamol) y de la prednisona (antiinflamatorio y antiagregante) en el curso de la GNMP, si bien los detalles de esta acción aún se desconocen.

Los hallazgos clínicos y de laboratorio citados antes ${ }^{13}, 16,27,28,38,41,44,50,74,81$, corfirman la participación de las plaquetas en la patogenia de las glomerulonefritis, ya sea que los mecanismos mediadores sean inmunológicos o no. Estos estudios demuestran que las alteracio. nes plaquetarias mejoran o se corrigen con el uso de aspirina, dipiridamol y prednisona ${ }^{13}, 14,16$, $17,27,28,33,39,41,44,50,66,69,75,81$, $104,112,116$. La aspirina y el dipiridamol inhiben la acción de enzimas plaquetarias como la ciclooxigenasa y la fosfodiesterasa respectivamente. Esta última disminuye la producción de tromboxano $\left(\mathrm{TXA}_{2}\right)$ una substancia de alta potencia vasoconstrictora y agregante de las plaquetas, por lo tanto predomina el efecto opuesto: vasodilatación $\mathrm{y}$ antiagregamiento plaquetario, debido a la persistencia de la síntesis de prostaciclina por las células de la pared arteriolar, con el consiguiente aumento del tiempo de sangria $y$ la ausencia o formación defectuosa de trombos. $\mathrm{La}$ antitrombosis producida por la aspirina está relacionada con la dosis de este medicamento. En dosis altas (por ejemplo, $200 \mathrm{mg} \mathrm{x} \mathrm{kg)} \mathrm{la} \mathrm{aspirina}$ es trombogénica en el animal experimental ${ }^{75}$; estudios "in vitro" demuestran que con las cantidades indicadas hay un aumento considerable de la adherencia de las plaquetas al endotelio, inducida por la trombina; el tejido arterial se hace protrombogénico. En humanos, la ingestión de $300 \mathrm{mg}$ de aspirina aumenta el tiempo de sangría mientras que diez veces esa dosis no demuestra este efecto. En realidad, la dosis alta bloquea el agregamiento plaquetario y la síntesis de $\mathrm{TXA}_{2}$ por aproximadamente 2 horas: Dos a tres días más tarde, se observa un aumento del tiempo de sangria y finalmente a los 6 a 7 días las alteraciones se normalizan, reflejando la estabilización de la sintesis de $\mathrm{TXA}_{2}$ y del agregamiento plaquetario. Por lo tanto, los cambios señalados parecen depender directamente de las concentraciones sanguineas de aspirina. De aquí que la manipulación del balance entre las acciones de $\mathrm{TXA}_{2}$ y $\mathrm{PGI}_{2}$ (prostaciclina) que regulan la función de las plaquetas circulantes "in vivo", determinaría la producción de estados pro o antitrombóticos de alta importancia clínica.

Los inhibjdores selectivos de la fosfodiesterasa (dipiridamol) serian, hasta cierto punto, de actividad más favorable que las dosis altas de aspirina. El dipiridamol permitiría que los endoperóxidos plaquetarios continúen siendo utiliza- 
dos por la pared yascular o por otras células manteniendo la síntesis de $\mathrm{PGI}_{2}$. Las dosis usuales o bajas de aspirina acetilan en forma irreversible la porción activa de la enzima por lo tanto, bloquean en foma permanente la sintesis de $\mathrm{TXA}_{2}$. Las plaquetas así afectadas no sintetizan proteina, de modo que una nueva ciclooxigenasa viene a reemplazar a la enzima inactiva sólo cuando se regeneran las plaquetas en la médula ósea y se entregan la circulación.

Los corticosteroides adrenales (cortisona y otros derivados) bloquean la sintesis de $\mathrm{PGl}_{2}$ al interferir con la liberación del ácido araquidónico por las células de la pared vascular 11,40. Estas hormonas, sin embargo, aumentan el número de plaquetas circulantes al estimular directamerte la médula ósea.

Basándonos en estos conceptos podríamos deducir, tentativamente, que la interpretación dada a los procesos inflamatorios humorales $y$ celulares conjuntamente con las alteraciones hemostáticas descritas, proporcionan una base racional para la aplicación de substancias antiagregantes de las plaquetas y de prednisona en la GNMP. La revisión de la literatura relacionada con la terapia de la GNMP revela que el uso de antiagregantes y de prednisona ha sido exitoso en un número importante de estos pacientes. Donadio y colaboradores 33,35 y West y McEnery69, 70, 110, 111 han publicado sus casuísticas recientemente. A pesar de que los resultados del estudio al azar y prospectivo presentado por Donadio son altamente llamativos, los aumentos de la creatinina sérica en el grupo control (sint tratamiento) comparada con la de pacientes que recibieron terapia antiagregante (aspirina y dipiridamol solamente), no fueron de significación estadística. Además, e] tratamiento no modificó la proteinuria, hematuria o los niveles de $\mathrm{C}_{3}$ plasmático. Estos investigadores postulan que la combinación aspirina-dipirida. mol disminuye la interacción entre las plaquetas y la pared vascular y el consumo (depósito y destruccion) plaquetario. Los autores indican también que las plaquetas de pacientes con GNMP transfundidas a individuos normales compatibles normalizan su vida media, en tanto que el experimento inverso revela una disminción significativa de la sobrevida de las plaquetas. Los hallazgos de Donadio y colaboradores coinciden con los de George y asociados 44 quienes demos. traron destrucción selectiva de las plaquetas, sin alteraciones de los niveles de fibrinógeno y plasminógeno en pacientes con GNMP. Los estudios de Donadio y colaboradores ${ }^{33}$ indican ausencia de correlación entre el consumo plaquetario y los niveles del complemento sanguíneo y sus depósito en el glomérub; la corre. lación fue estrecha, sin embargo, entre la destrucción de plaquetas e inflamación o proliferación celular glomerular. Importante evidencia adicional ha sido proporcionada recientemente por Clark $^{27}$ y colaboradores quienes determinaron que las plaquetas son mediadoras del daño tisular (renal) y se consumen y depositan activamente en el glomérulo. Más aún, la inyección de adenosina difosfato en el conejo produce la formación de microtrombos plaquetarios los que se asocian a glomerulonefritis, glomeruloesclerosis e hipertensión arterial. ${ }^{57}$

El uso de aspirina y dipiridamol en la serie de Donadio y colaboradores ${ }^{3} 3$ produjo sangramiento importante en 3 pacientes en los cuales el tratamiento debió ser suspendido. Otros, utjlizando estos agentes 25,112 , no han observado sangramiento serio en los pacjentes. Nuestras propias observaciones en niffos indican que cuando aspirina y dipiridamol se utilizan en los dosis recomendadas corregidas por $\mathrm{Kg}$ de peso (13 y $3 \mathrm{mg}$ respectjvamente) ocurren frecuentemente sangramientos minimos o hematomas cutáneos. Es difícil establecer si individuos (niños $\mathrm{y}$ adolescentes) cuya actividad física es considerable, desarrollarán sangramientos de importancia como complicación de esta terapia. Sin embargo, mientras esta posibilidad exista recomendamos una dosis menor que la citada anteriormente. Los estudios de West, McEnery y McAdams 69, 70. $94,110,111$ muestran, comparados con otros 2 . $30,46-48$, el porcentaje mas alto de sobrevida y el menor número de complicaciones publicadas hasta la fecha. Estos autores han usado prednisona por vía oral en dosis única de $2 \mathrm{mg} \mathrm{x} \mathrm{kg} \mathrm{x}$ día (máximo de $80 \mathrm{mg}$ ) cada 48 horas desde 1964; el tratamiento fue continuado por un perjodo mínimo de 2 afios al término del cual se realizó una segunda biopsia renal66,69,70. La dosis de prednisona mencionada antes fue subsecuentemente reducida 0 , en todo caso, se redujo en términos relativos a la masa corporal debido al aumento de peso de los pacientes, cuya mayoría continuó creciendo en forma satisfactoria. Otros efectos colaterales fueron mínimos. Estos investigadores enfatizan además un problema clínico importante, cual es la reaparición de ta GNMP meses 0 años después de suspendido el tratamiento. Por lo tanto, recomiendan una dosis de mantención permanente de aproximadamente 15 a $20 \mathrm{mg}$ de prednisona en dosis oral única día por medio. Con este régimen han sido tratados 66 pacientes, 48 de los cuales han sido seguidos hasta por 20 años. Cinco casos (10.4\%) desarro. llaron IRT y la sobrevida total del grupo es de $80 \%$ a los 18 años de seguimiento. ${ }^{11}$ Mientras $91 \%$ de los pacientes tenian hipocomplementemia al comienzo de la terapia, el análisis de la 
población en 1984 demostró que $93 \%$ de los casos tenían complementemia normal. La crea. tinina sérica era menor de $3 \mathrm{mg} x$ dl en $90 \% \mathrm{y}$ menor de $1,6 \mathrm{mg} x \mathrm{dl}$ en $86 \%$ de los pacientes. La información proporcionada por estos estudios es, a nuestro juicio, de gran importancia clínica ya que indica en forma evidente que la prednisona constituye uno de los medicamentos de elección en el tratamiento de la GNMP.

Los parámetros clínicos más útiles para juzgar la mejoría del paciente son: normocomplementemia persistente en un enfermo previamente hípocomplementémico; desaparición de la actividad lítica del plasma (factor nefrítico) para el $\mathrm{C}_{3}$ en especial en portadores de GNMP Tipo 2; disminución de la severidad de la histopatológia en una segunda biopsia renal; normalización del sedimento urinario; desaparición del sindrome nefrótico; mantención de la creatinina sérica en niveles normales o su reducción a la normalidad y finalmente, cono corolario de lo anterior, normalización de la filtración glomerular. La evaluación de la literatura publicada indica claramente que sólo en los pacientes tratados con prednisona en la forma descrita se han logrado algunos o la mayoría de los efectos indicados. Sin embargo, la combinación aspirina-dipiridamol también "ha proporcionado por primera vez evidencia convincente que los agentes antiagregantes plaquetarios son beneficiosos en la glomerulonefritis humana". 16 Por lo tanto, a nuestro juicio existe amplia justificación en la actualidad, para utilizar de rutina la combinación pređinisona-aspirinadipiridamol por un tiempo prolongado, en pacientes pediátricos y adultos con GNMP, independientemente de su tipo histológico. Estudios colaborativos de esta enfermedad y el uso de protocolos terapéuticos estandarizados a nivel nacional $e$ internacional, son altamente necesarios para demostrar en forma cuantitativa, sin ambiguedades, la validez de estos medicamentos, que por el momento parecen prometer el control más adecuado de la inflamación glomerular progresiva y lo que es aún más interesante, de la glomerulosclerosis en general.

\section{RESUMEN}

La GNMP, una entidad crónica y progresiva, frecuente en niños y adultos, produce insuficiencia renal terminal en un número significativo de pacientes. Estudios inmunohistopatológicos permiten distinguir las variedades 1 y 2 y posiblemente una tercera, cuyas manifestaciones clínicas particularmente en el niño menor son indistin. guibles $y$ en algunos enfermos semejan una GN difusa aguda de etiología estreptocócica o un síndrome nefrótico idiopático. En estos casos se requieren determinaciones seriadas del complemento sérico $\left(\mathrm{C}_{3}\right.$ y $\left.\mathrm{C}_{4}\right)$ y biopsia renal para el diagnóstico diferencial clínico y tipificación histológica.

Debido a su progresividad, se han ensayado numerosos regimenes terapéuticos. En la actua. lidad, los estudios que demuestran la participación de las plaquetas y de las células mononucleares en el proceso destructivo glomerular proporcionan un trasfondo racional para el empleo de medicaciones inhibidoras de la agregación y activación plaquetaria (aspirina $y$ dipridamol) y antiinflamatorios de alta potencia como la prednisona. Estas substancias, usadas en conjunto diariamente y en forma alternada, respectivamen. te, en regímenes prolongados ( 3 a 5 años o más) e iniciados a los pocos meses de hecho el diag. nóstico parecen haber logrado un control efectivo de la progresión de la glomerulopatía sin grandes efectos nocivos debidos al tratamiento. Su uso combinado ha aumentado significativamente la sobrevida de pacientes pediátricos y adultos en series clínicas publicadas recientemente. Es nuestra convicción que el tratamiento combinado descrito (aspirina-dipridamolprednisona) es la terapia de elección en la GNMP independientemente de su tipo histológico y que otros tratamientos o la ausencia de este no es justificable.

\section{REFERENCIAS}

1. Anders, D., Agricola, B. Sippel, M., Thoenes, W.. Basement membrane changes in membranoproliferatjve glomerulonephritis. Virchow's Arch. Path. Anat. 37: 1, 1977 .

2. Antoine, A., Foye, C.: The clinical course associated with dense deposits in the basement membrane. Kidney Int. 1; 420, 1972.

3. Barbiano di Belgiojow, G, Tarantino, A., Bazzi, C., Colasanti, G. Guerra, W., Durante, A,: Immunofluorescence patterns in chronic membranoproliferative glomerulonephritis (MPGN). Clin. Nephrol. 6: 303, 1976.

4. Bartlow, B.G., Roberts, J.K., Lewis, E.J.: Nonimmunoglobulin $C_{3}$ activating factor in menbra noproliferative glomerulonephritis. Kidney Int. 15: 294, 1979.

5. Bennetf, W.M., Bardana, EJ., Wuepper, K., et al, Partial lipodystrophy, $C_{3}$ nephritic factor and clinically inapparent mesangiocapillary glomeIulonephritis. Am. J. Med. 62: 757, 1977.

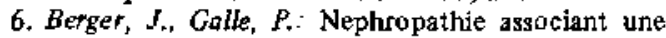
glomerulite proliferative et de dépots au sein de basales. I Etude anatomo-clínique de 13 cas. J. Urol. Nephrol. 69: 90, 1963.

7. Berger, J., Galle, P.: Alteration singuliere des membranes basales du rein. J. Urol. Nephrol 68: $116,1962$.

B. Berger, I., Galle, P.: Depots denses au sein de membranes basale du rein. Etudes en microsoopies optique et electronique. Presse Med. 49: 2351, 1963. 
9. Berry, P.L., McEnery, P.T, McAdams, A.J., West, C.D.: Membranoproliferative glomerulonephritis in two sibships. Clin. Nephrol, 16: 101, 1981.

10. Berthoux, F.C., Ducret. F, Colon, S., BlancBrunat, N., Zech, P.Y., Taeger, J.: Renal trans. plantation in mesangiopsoliferative glomerulonephritis (MPGN): Relationship between the high frequence of Iecurrent glomerulonephritis and hypocomplementemia. Kid. Int, 7: S-323, 1975.

11. Blajchman, M.A., Senyi, A.F., Hirsh J, Buchanan, M., Mustand, J.F.: Shortening of the bleeding time in rabbits by hydrocortisone caused by inhibition of Prostacyclin generation by the vessel wall. J. Clin. Invest. 63: 1026, 1979.

12. Bohle, A., Gartner, H.U., Fischbach, H., Bock, $K . D .$, et al.: The morphologic and clinical features of membranoproliferative glomerulonephritis in adults. Virchows Arch. Path. Ant. 363: 213, 1974 .

13. Born, G.V.R.: Mechanisms of intravascular platelet aggregation in renal diseases. In "Hemostasis, Prostaglandins and Renal Disease". Editors: Renuzzi, G., Mecca, G., de Gaetano, G., Raven Piess, NY. 1980, p. 21.

14. Bunag, R.D. Douglos, R.C. Imai, S., Berne, R.M.: Influence of a pyrimidopyrimidine derivative on deamination of adenosine by blood. Circ. Res. XV: $83,1964$.

15. Camerón, J.S., Glasgow, E.F., Ogg, C.S.. White, R.H.R.: Membranoproliferative glomerulonephritis and persistent hypocomplementemia, Brit. Med. J. 4: 7,1970 .

16. Camerón, J.S.: Phatelets in glomerular disease, Ann. Rev. Med. 35: 175, 1984.

17. Camerón, J.S.: Glomerulonephritis: Current problems and understanding. J. Lab. Clin. Med. 99: 755,1982 .

18. Camerón, J.S., Turner, D.R., Heaton J., et al : Idiopathic meangiocapillary glomerulonephritis. Comparison of types $I$ and $I I$ in children and adults and long-term prognosis. Am. J. Med. 74 : $175,1983$.

19. Camerón, J.S. Ogg, C.S., White, R.H.R., Glasgow. E.F.: The clinical features and prognosis of patients with normocomplementemic mesangiocapillary glomerulonephritis. Clin Nephrol. 1: 8, 1973.

20. Camerón, J.S., Vick, R.M., Ogg, C.S., Syemour, W.M., Chantier, C., Turner D.R.: Plasma $C_{3}$ and $\mathrm{C}_{4}$ concentrations in management of glomerulonephritis. Brit. Med. J. 7: 668, 1973 (23 September).

21. Camerón, J.S.: Mesangiocapillary glomerulonephritis. In: Straus, J. ed. The nephrotic syndrome. Pediatric Nephrology, Vol. 5, New York, Garland Press; 1179, 153.

22. Carlo, J., Ruddy, S., Sauter, S., Yount, W.J.: Deposition of $1 \mathrm{H}$ globulin in kidney of patients with immune renal disease. Arthr. Rheum. 22: 403,1979 .

23. Catram D., Charron, R, Cordella, C, et al: Controlled trial in mesangiocapillary glomerulonepluitis (MCGN) (abstr) In: Zurukzogh, W., Papadimitriou, M., Sion, M. et al. eds 8th International Congress of Nephrology: advances in basic and clinical nephrology. Basel, Karger, 1981: 287.

24. Chapman, S.J., Comeron, I.S., Chantler, C.. Tumer, D.: Treatment of mesangiocapillary glomerulonephritis in children with combined immunosuppression and anticoagulation. Arch.
Dis. Child. 55: 446, 1980.

25. Chesebro, J.H., Clements, J.P., Fuster, $V_{+}$et al : A platelet-inhibitor-drug trial in coronary-artery bypass operations: Benefit of perioperative dipyridamole and aspirin therapy on early post-operative vein-graft patency. N. Engl. J. Med, 307: 73, 1982.

26. Churg, $J_{+}$, Duffy, J.L., Bernstein, J.: lient ification of dense deposit disease. A report for the international study of kidney disease in children. Arch. Patho. Lab. Med. 103: 67, 1979.

27. Cork, W.F., Friesen, M., Linton, A.L., Linsay. $R . M$.: The platelet as a mediator of tissue damage in immune complex glomerulonephritis. Clin. Nephrol. 6: 287, 1976 .

28. Clark, W.F., Lewis, M.L., Cameran, J.S., Parsons, $V .:$ Intrarenal platelet consumption in the diffuge proliferative glomerulonephritis of systemic lupus erythematosus. Clin. Sci. Mol Med. 49: 247, 1975.

29. Curtis, J.J., Wyatt, R.J. et al: Renal transplantation fot patients with type I and type Il membranoproliferative glomerulonephritis Am. J. Med. 66: $216,1979$.

30. Davis, A.E.. Schneeberger, E.E. et al : Membranoproliferative glomerulonephritis (MPGN type I) and dense deposit disease (DDD) in children. Clin. Nephrol. 9: 184, 1978.

31. Dov's, C.A., Marder, H., West, C.D.: Circulating immune complexes in membranoproliferative glomerulonephritis. Kidney Int. 20: 728, 1981.

32. Davis, A.E., Schneeberger, E.E., McCluskey, R.T., Grupe, WE.: Mesangial proliferative glomerulonephritis with irregular intramembranous deposits. Another variant of hypocomplementemic nephritis. Am. J. Med. 63: 481, 1977.

33. Donadio, J.V., Anderson, C.F. Mitchell, J.C. III, ef $a l$ : Membranoproliferative glomerulonephritis. A prospective clinical trial of plateletinhibitor therapy. Engl. J. Med. 310: 1421, 1984.

34. Donadio, J.V. Jr., Hollwy, D.E.: Membranoproliferative glomerulonephritis. Semin. Nephrol. 2: 214,1982 .

35. Donadio, J.Y., Slack, T.K., Holley, X., Mstrup, $D . M$. Idiopathic membranoproliferative (mesangiocapillary glomenulonephritis): A clinicopathologic study. Mayo Clin Proć. 54: 141, 1979.

36. Dreher, W.H., Zimmerman, S.W., Simpson, D.P.: Hyperchloremia associated with membranoproliferative glomerulonephritis. Nephron 18: 321, 1977.

37. Droz, D., Zanetti, M., Noel, L. - H., Leibawitch, J.: Dense deposit disease. Nephron 19: 1, 1977.

38. Duffus, P., Parbtani, A, Frampton, G., Cameron, $J . S$ : Intraglomerular localization of platelet-re. lated antigens, platelet factor 4 and -thromgoglobulin in glomerulonephritis. Clin. Nephrol. 17: 288,1982 .

39. Fleischmon, A.I. Bierenbaum, M.L., Sitler, A.: The effect of aspirin on in vivo platelet function in humans. Thromb. Res. 8: 797, 1976.

40. Flower, R.I.: "Advances in Prostaglandin and Thromboxane Research", 1978, Vol. 3, pp. 105, edited by R. Paoletti and B. Samuelson, Acadernic Press, New York.

41. Fuster, $V_{\text {, }}$ Chesebro, J.H.: Antithrombotic therapy: Role of: platelet-inhibitor drigs. Phar. macologic effects of platelet-inhibitor drugs. Mayo Clin. Proc. $56185,1985$.

42. Galle, P.: Mise év évidence au microscope électro- 
nic d'une lésion singuliére des membranes basales du rein et de la substance hyaline (Thesis; quoted in Droz et al. Nephron 19: 1, 1977.

43. Geiger, H., Good, R.A., Day, N.K.: Clearances of complement components, $C_{3}$ proactivator and other serum proteins in chronic MPGN. Clin. Nephrol 3; 139, 1975.

44. George, C.R.P. ef al: A-kinetic evaluation of hemostasis in renal disease. N. EngL J. Med. 291 : 1111,1974 .

45. Goroff, S.P., Fellers, X.F., Vowter, G.F., Haneway, C.A., Rosen, F.S.: The $1 \mathrm{C}$ globulin in childhood nephrotic syndrome: laboratory diagnosis of progressive glomerulonephritis. N. Engl. J. Med. 273: 524, 1965.

46. Habib, R., Kleinknecht, C., Gubler, M.C., Leyy, M.: Idiopathic membranoproliferative $G N$ in children: Report of 105 cases. Clin. Nephrol. 1: 194, 1973.

47. Habib, R, Levy, M.: Clinicopathologic correlations in 128 cases of MPGN. Proc. 6 th Int. Congr. Nephrol., Florence, 1975, pp. 462. Karger, Basel 1976.

48. Habib, R., Gubler, M.C., Loirat, C.,Ben-Mriz, H., Levy, $M$.: Dense deposit disease: A variant of membranoproliferative giomerulonephritis. Kidney Int. 7: $204,1975$.

49. Holbwachs, L., Leveillé, M., Lesaire, P.L., Wattell, S., Leibowitch, J.: Nephritic factor of the classical pathway of complement. J. Clin. Invest. 65:1249, 1980.

50. Hayslett, J.P.: Role of platelets in glomerulonephritis. N. Engl J. Med. 310: 1457, 1984 .

51. Herdmen, R.C., Pickering, R.J., Michael, A.F., Vemier, R.L., Fish, A.J., Gewurz, H., Good, RA.: Chronic glomerulonephritis associnted with low serum complement activity (chronic hupocomplementemic glomerulonephritis) Medicine 49: 207, 1970 .

52. Holland, N.H., De Bracco, M.M.E., Christian, C.L.: Pathways of complement activation in glomeruJonephritis. Kidney Int. 1: 106, 1972.

53. Hymon. L., Jenis, E.H., Hill, G.S., et al: Alternate pathway activation in pneumacoccal glomerulanephritis. Am. J. Med. 58: 810, 1975.

54. International Study of Kidney Diseoses in Children: Alternate-day steroid therapy in membranoproliferative glomerulorephritis: A randomized contral clinical trial (A bstr) Kid ney Int. 21: 150,1982 .

55. Jeune, M., Freycon, M.T., Hermier, M. et al: Les nephropathies dans la lipodystrophie "progressive ou maladie de Barraquer-Simons, Ann. de Pediatrie 50: $2869,1965$.

56. Jones, D.B.: Membranoproliferative glomerulonephritis. One or many diseases. Arch. Pathol. Lab. Med, 101: 457, 1977.

57. Jorgensen, L., Giynn, M.F, Houg, T., Murphy, E.A., Buchanan, M.R., Mustard, J.F.: Renal lesions and rive in blood pressure caused by adenosine diphosphate-induced phatelet agpregation in rabbits. Lab. Inves 23: 347, 1970.

58. Kim, $Y_{r,}$ Michael, A.F.: Idiopathic membranoproliferative glomerulonephriti. Annu. Rev. Med. 31: $273,1980$.

59. Kin, Y., Vernier, R.L., Fish, A.J., Michael, A.F.: Immunofluorescence studies of dense deposit disease: The presence of railroad tracks and mesangial rings. Lab. Invest. 40:474, 1979.

60. Kincoid-Smith, $P$ : The treatment of chronic meangiocapillary (membranoproliferative) glo- merulonephritis with impaired renal function. Med. J. Australia 2: 587,1972 .

61. King, J.R., Valenzuela, R., McCormack, L.J.. Osbom, $O, G .$. Granular dense deposit disease. Iab. Invest, 39: 591, 1978.

62. Klein, M., Poucell, S., Arbus, G.S., McGraw, M., Rance, C.P., Yoon, S.-J., Baumol, R.: Characteristics of a benign subtype of dense deposit disease: Comparizon with the progressive form of the disease. Clin. Nephrol. 20: 163, 1983.

53. Lamb, V., Tisher, C.C., McCoy, R.C., Robinson, $\boldsymbol{R} . \boldsymbol{R}$ : Membranoproliferative glomerulonephritis with dense intramembranous alterations. A clinjcopathologic study. Lab. Invest. 36: 607, 1977.

64. Levy, M., Gubler, M.C., Sich, M., Beziau, B., Habib, R.: Immunopathology of membranoproliferative glomerulonephritis with subendothelial deposits (Type 1 MPGN). Clin. immunol. Immun. Path. 10: 477, 1978.

65. Mahieu, P.: Biochemical structure of glomerular basement membrane in chronic glomerulonephritis. I. Globular and membranoproliferative glomerulonephritis. Kidney Inst. 1: 115, 1972.

66. McAdoms, A.J., McEnery, P.T. Wer, C.D.: Mesangiocapillary glomerulonephritis: Changes in glomerular morphology with long-term alternate-day prednisone therapy. J. Pediat. 86: 23, 1975.

67. McAdams, A.J.: The glomerular morphology of membranoproliferative glomerulonephritis (MPGN). Proc. 5th Int. Pediat. Nephrol. Symposium. 1981, Gruskin, A.B., Norman, M.E.: editors. Martinus Nijhoff, Boston, p. 340.

68. HcCoy, R.C., Clapp, J, Seigler, H.F.: Membranoproliferative glomerulonephritis Am. J. Med. 59: 288, 1975,

69. McEnery, P.T., McAdams, A.J., West, C.D.: Membranoproliferative glomerulonephritis: Improved survival with alternate-day prednisone therapy. Clin. Nepurol. 13: 117, 1980.

70. McEnery, P.T. Strife, C.F.: Chronic glomerulonephritis in children, In: Pediatric Nephrology. Time B.M., Mendoza Sa, Brenner M.B., Steion J.H., editors, 1984, pp. 231, Vol. 12, Churchill, Livingstone, New York.

71. McIntosh, R.M.. Griswola, W.D., Smith, F.G., Kaufman, D.B., Urizor, R.E., Vernier, R.L.: Azathioptine in GN. Lancet I: $1085,1972$.

72. McLean, R.H., Siegel, N.., Kashgorian, M.: Activation of the classic complement pathway in patients with the $\mathrm{C}_{3}$ nephritic factor. Nephron 25 : 57,1980 .

73. McLean, R.H, Michael, A.F..' Properdin and $C_{3}$ proactivator: Alternate pathway components in human glomerulonephritis. J. Clin. Invest. 52: $634,1973$.

74. Miller, K., Dresner, I.G., Michael, A.F.: Localization of platelet antigens in human kidney disease. Kidney Int. 18: 472, 1980.

75. Moncada, S., Korbut, R.: Dipyridamole and othet phosphodisterase inhibitors act as antithrombotics agents by potentiating endogenous prostacycline. Lancet 1: 1286, 1978.

76. Monga, G., Mazzuco, G., Barbiana di Belgiojoso, G., Busnach $G$.: The presence and possible role of monocyte infiftration in human chronic proliferative glomerulonephritis Am. J. Pathol. 94: 271, 1979.

77. Myers, B.D., Griffel, B., Naveh, D., Jonkielawita, T., Klajmen, A.: Membranoproliferative glomenlonephritis associated with persistent viral 
hepatitis. AJPC 59: 222, 1973,

78. Northway, J.D., McAdams, A.J., et al: A "silent" phase of hypocomplementemic persistent nephritis detectable by reduced serum $1 \mathrm{C}$ globulin levels. J. Pediat. 74: 28, 1969.

79. Olson, J.L., Philips, T.M., Lewis, M.G., Solez, K.: Malignant melanoma with renal dense deposits containing tumor antigens. Clin. Nephrol. 12: 74, 1979.

80. Oot, V.M., Vallota, E.H., West, C.D.: Serum immune complexes in MPGN and other glomeulonephritides. Kidney Int. 11: 275, 1977.

81. Parbtani, A., Cameron, J.S.: Platelets involvement in glomerulonephritis In "Hemostasis," Prostaglandins and Renal Disease". Remuzzi, G., Mecca, G., de Gaetano, G.: editors, Rayen Press, NY. 1980 , p. 45.

82. Peters, D.K., Mortin, A., Weinstein, A. et al: Complement studies in membranoproliferative glomerulonephritis. Clinical and Exper. Immunol. 11: $311,1972$.

93. Pickering, R.J., Gewurz, H. Good, R.A.: Complement inactivation by serum from patients with acute and hypocomplementemic chronic glomerułonephritis. J. Lab, Clin Med.

84. Pickering, R.J., Michael, A.F., Herdmon, R.C., Good, R.A., Gewtur, H.: The complement system in chronic glomerulonephritis: Three newly associated aberrations. J. Pediat. 78: 30, 1971.

85. Royer, P., Habib, R. Vermeil, G., Mathieu, H., Alizon, $M$.: Les glomerulonephritis prolongees de l'infant. Ann. Pediat. 38: 173, 1962.

86. Rudofsky, U.H., Urizar, R.E., Gabrielsen, AE, et al: Dactinomycin treatment of murine lupus erythematosus I. Renal disease and longevity. Lab. lnvest. 39: 441, 1178.

87. Chena, F.P., Pertosa. G., Stanzigle, P., Vox, E., Pecoraro, C., Andreucci, V.E.: Biological significance of the $C_{3}$ nephritic factor in membranoproliferative glomerulonephritis. Clin. Nephrol, 18: 240,1982 .

88. Schifferli, J.A.: Complement in Nephritis. In "Glomerular injury 300 years after Morgagni". Bertani, T., Remuzzi, G.: editors. Wichtig Editore, Milán, 1983, p. 57.

89. Scott, D.M., Amos, N., Sissons, J.G.P., Lachman, P.J, Peters, D.K.: The immanoglobulin nature of nephritic factor (NeF). Clin. Exp. Immunol, 32: $12,1978$.

90. Sissons, J.G.P., West, R.J., Fallows, I., Williams, D.G., Boucher, C.J., Amos, N., Peters, D.K.: The complement abnormalities, of lipodystrophy. $\mathbf{N}$. Engl. J. Med. 294: 461, 1976.

91. Strife, C.F., McAdoms, AJ., West, C.D.: Membranoproliferative GN characterized by focal segmental proliferative changes. Clin. Nephrol. 18; 9, 1982 .

92. Strife, C.F., Jackson, E.C., McAdams, A.J.: Type IIl MPGN: Long-term clinical and morphologic evaluation. Clin. Nephrol, 21: 323, 1984.

93. Strife, C.F., Hug, G., Chuck, G. et al: Membranoprobiferative glomerulonephritis and $\alpha$ 1-Antitrypsin deficiency in children.Pediatrics 71: 88,1983

94. Sirife, C.F., McEnery, P.T., McAdoms, A.J., West, C.D.: Mombranoproliferative glomerulonephritis with disruption of the glomerular basement membrane. Clin. Nephrol. 7: 65, 1977.

95. Sweinson, C.P., Robson, J.S., et al: Mesangiocapillary glomerulonephritis. $A$ long-term study of
40 cases. J. Pathol 141: 449, 1983.

96. Tiller, D.J., Clarkson, A.R., Mathew, T., et al, A prospective randomized trail in the use of cyclophpsphamide, dipyridamole and warfarin in membranous and mesangiocapillary glomerulonephritis. In: Zurukzoglu, W., Papadimitriou, M., Sion, M. at ar: eds. Advarces in Basic and Clínical Nephrology. Basel, S. Karger 346, 1981.

97. Thompson, R.A.: $\mathrm{C}_{3}$ inactivating factor in the serum of a patient with chronic hypocomplementemic proliferative glomerulonephritis. Immuno$\operatorname{logy} 22: 147,1972$.

98. Thompson, $R, A: \operatorname{lgG}_{3}$ levels in patients with chronic membranoproliferative glomerulonephritis. Brit. Med. J. 1; 282, 1972.

99. Urizar, R.E., Tinglof, B., McIntosh, R.M., Litman, N.L. Bamett, E., et al: Immunosuppressive therapy of proliferative GN in children. Am. J. Dis. Child. 118: 411, 1969.

100. Urizar, R.E.: The hemolytic uremic syndrome, In: Pediatric Nephrology: New Directions in Therapy. Medical Examination Publishing Co. Inc., New Hyde Park, NY., 1983, pag. 250.

101. Urizar, R.E., Gilboa, N., Largent, JA.: Immune mechanisms of childhood renal diseases. Paediatrician 10: 254, 1981.

102. Urizar, R.E., Herdman, R.C.: Hypocomplementemic glomerulonephritis, In: Pediatric Nephrology, Rubin, M., Barratt, M., editors. Williams and Wilkins, Baltimore, 1975 , pp. 569.

103. Vallote, E.H., Forristal, J., Spitzer, R.E., et al: Continuing $\mathrm{C}_{3}$ breakdown after bilateral nephrectomy in patients with membranoproliferative glomerulonephritjs. J. Clin. Invest. 50: 552, 1971 ,

104. Vanrenterghem, Y., Roels, L., Verberckmoes, $R$., Michielsen, P.: Treatment of chronic glomerulonephritis with a combination of indomethacin and cylophosphamide. Clin. Nephrol 4: 218, 1975.

105. Vargas, R., Thompson, X.J., Wilson, D., Camerón, $J . S$. et al: Mesangiocapillary glomerulonephritis with "dense" deposits in the basement membranes of the kidney. Clin. Nephrol. 5: 72, 1976.

106. West, C.D. et al: Hypocomplementemic and normocomplementemic persistent (chronic) GN; clinical and pathologic characteristics. J Pediatr. 67: $1089,1965$.

107. West, C.D., McAdams, A.J.: Serum 1C globulin levels in persistent GN with low serum complement, variability unrelated to clinical course. Nephron $7 ; 193,1970$.

108. West, C.D., Winter, $S_{\text {, }}$ Forristat, $J$, et al: Evidence for in vivo breakdown of $1 \mathrm{C}$-glogulin in hypocomplementemic glomerulonephritis. J. Clin. Invest. 46:5389, 1967 .

109. West, C.D.: Hyomplementemia in glomerulonephritis. South. Med. J. 68: 1194, 1975.

110. West, C.D.: Membranoproliferative glomerulonephritis: Classification and treatment. In Pediatric nephrology. Proceedings of the Fifth International Pediatric Nephrology Symposium, 1981, Gruskin, A.B., Normart, M.E.: editors. Martinus Nijhoff, Boston, p. 108 .

111. West, C.D.: Personal Communication (1984).

112. West, J., Bellet, S., Manzoli, U.C., Müller, O.F.: Effects of persantin (RA8) a new coronary vasodilat or on coronary blood flow and cardiac dynamics in the dog. Circul. Res X: 35, 1962.

113. Whaley, $K$.. Ruddy, S.: Modulation of the altexnative complement pathway by $1 \mathrm{H}$ globulin. J. Exp. Med. 144: 1147, 1976.

114. Williams, D.G., Lachman, P.J., at al: Role of $\mathrm{C}_{3} \mathrm{~b}$ 
in the breakdown of $C_{3}$ in hypocomplementemic mesangiocapillary glomerulonephritis. The LANCET: 1 (\#801) 447, 1973.

115. Zamurovic, D.. Churg, J.: Idjopathic and secondary mesangiocapillary glomerulonephritis. Nephron 38: $145,1984$.

116. Zimmerman, S.E., Moorthy, A.V., et al: Prospective trial of Warfarin and Dipyridamole in patients with membranoproliferative glomerulonephritis. Am. J. Med. 75: 920, 1983.
117. Zimmermon, S.W., Hyman, L.R., Vehling, D.T., Burkholder, P.M.: Recurrent membranoproliferative glomerulonephritis with glomerular properdin deposition in allograft. Ann. Int. Med. 80: $169,1974$.

118. Zuccheli, P., Sasdelli, M., Cagnoli, L., Donini, V., Cosanova, $S$, Rovinetti, C.: Membranoproliferative glomerulonephritis: Correlations between immunological and histological firidings. Nephron 17: 449, 1976. 